\title{
OPTIMIZING TERRESTRIAL LASER SCANNING MEASUREMENT SET-UP
}

\author{
Sylvie Soudarissanane and Roderik Lindenbergh \\ Remote Sensing Department (RS)) \\ Delft University of Technology \\ Kluyverweg 1, 2629 HS Delft, The Netherlands \\ (S.S.Soudarissanane, R.C.Lindenbergh)@tudelft.nl \\ http://www.lr.tudelft.nl/rs
}

Commission WG V/3

KEY WORDS: Laser scanning, point cloud, error, noise level, accuracy, optimal stand-point

\begin{abstract}
:
One of the main applications of the terrestrial laser scanner is the visualization, modeling and monitoring of man-made structures like buildings. Especially surveying applications require on one hand a quickly obtainable, high resolution point cloud but also need observations with a known and well described quality. To obtain a 3D point cloud, the scene is scanned from different positions around the considered object. The scanning geometry plays an important role in the quality of the resulting point cloud. The ideal set-up for scanning a surface of an object is to position the laser scanner in such a way that the laser beam is near perpendicular to the surface. Due to scanning conditions, such an ideal set-up is in practice not possible. The different incidence angles and ranges of the laser beam on the surface result in 3D points of varying quality. The stand-point of the scanner that gives the best accuracy is generally not known. Using an optimal stand-point of the laser scanner on a scene will improve the quality of individual point measurements and results in a more uniform registered point cloud. The design of an optimum measurement setup is defined such that the optimum stand-points are identified to fulfill predefined quality requirements and to ensure a complete spatial coverage. The additional incidence angle and range constraints on the visibility from a view point ensure that individual scans are not affected by bad scanning geometry effects. A complex and large room that would normally require five view point to be fully covered, would require nineteen view points to obtain full coverage under the range and incidence angle constraints.
\end{abstract}

\section{INTRODUCTION}

The available mid-range 3D Terrestrial Laser Scanners capture the 3D positions of a scenery with data acquisition of unprecedented speed, with accuracies in the order of millimeters. The scanner provides a 3D visualization of a scene by measuring distances to object surfaces in a spherical coordinate system. For each point, it records a horizontal and a vertical angle, and a range measurement from the hit point on the surface to the scanner, with the center of the scanner as the origin of a local coordinate system. The reflection of the laser beam on the object surface is used to acquire a range measurement as well as an intensity value of the reflected light (Vosselman and Maas, 2010). Although the point measurements are accurate, scans are subject to measurement noise. Manufacturers often provide technical specifications including accuracy of measurements performed on reference surfaces under laboratory conditions (Faro, 2010; Leica, 2010). Manufacturers accuracy specifications are often set without regard to the inherent variability that exists in the hardware (Dorninger et al., 2008; Kersten et al., 2004; Lichti, 2007), in the atmospheric conditions (Hejbudzka et al., 2010; Borah and Voelz, 2007; Hoefle and Pfeifer, 2007; Voisin et al., 2007), in the object properties (Kaasalainen et al., 2009; Bucksch et al., 2007) or in the scanning geometry (Soudarissanane et al., 2011; Schaer et al., 2007).

Systematic and random errors of individual point measurements propagate through standard processing steps to the final products like $3 \mathrm{D}$ as built models or structural monitoring results. In Soudarissanane et al. (2011), an original approach is presented to model the scanning geometry effects by focusing on the incidence angle and the distance to an object's surface. It is shown that by considering the influence of the distance on the signal to noise ratio, the increase in measurement noise with increasing incidence angle and range could be successfully modeled.

Several scans taken from different view points are needed to have a full coverage of the object surface and to avoid major occlusions. Under stable atmospheric conditions, large variability is observed in individual points produced during surveys consisting of several scans taken from different locations around an object. In practice, most scenery contains a large variety of materials and different parts of the scenery are scanned with a different scanning geometry from several view points. The Terrestrial Laser Scanner is a remote sensing technique commonly used for more than a decade in a wide range of engineering applications, such as geodetic survey, civil engineering, forestry, forensics or cultural heritage. Despite the large variety of applications, the optimal view point placement for an accurate and reliable acquisition has not yet been investigated. In this paper, it is shown how scanning geometry constraints can be exploited to improve the measurement set up. By defining an optimal scanner view point in the scenery, a spatial coverage of good quality is ensured. Moreover, the definition of the minimum number of view points needed to cover an object or an area is investigated. This optimal measurement set up definition allows the acquisition of accurate data that can be further processed with a smaller propagation of errors.

\section{PLANNING VIEW POINT POSITIONS}

Multiple scans acquired from different view points are required to sample all visible surfaces of an unknown scene. In this paper, we define a view point as the location of the center of the scanner in the scene. Each scan consists of 3D points relative to the center of the scanner. From a view point the surfaces in the line of sight of the scanner are defined as visible. A scene is defined in three dimensions and can be represented using e.g. vertexes, 
polygons or tetrahedrons. Almost all 3D indoor scene can be reduced to a 2D map by taking an horizontal cross section of the scene at for instance the height of the sensor. This approximation of the 3D surrounding as a 2D map results in less intensive computations. Moreover, this 2D map may include holes that can be caused by e.g. walls, furniture in the room, columns. The horizontal cross-section enables to visualize a floor map at the height of the scanner, with holes consisting of non-transparent objects in the scene, as depicted in Fig. 1.

Local sequence of scans obtained to cover an entire 3D scene often yield in abundant number of scans, in most cases redundant, with too many overlapping areas or with bad scanning geometry. As each acquisition costs a significant amount of time, a minimum number of scan locations is desired to cover the scene. Moreover, post-processing steps such as the registration are affected by the number of acquisitions in a scene. On one hand, too little acquisitions and far apart might result in scans that cannot be registered due to insufficient overlap. On the other hand, too many scans are computationally intensive to register in one common coordinate frame. A trade-off between the number of acquisitions and the computational efforts needs to be defined. In the past years, several point cloud registration methods have been developed. Existing approaches to register point clouds are based on two main techniques. The first technique incorporates tie points that are common in two point clouds, and estimates transformation parameters by minimizing the distance between the corresponding tie points (Besl and McKay, 1992). The second technique relies on the adjustment of corresponding surface geometry based on surface normal estimation (Gruen and Akca, 2005; Gorte et al., n.d.; Vosselman et al., 2004; Khoshelham and Gorte, 2009). In both approaches, samples of the surface need to be acquired with good quality to estimate a tie point location or a surface normal. The registration quality is highly dependant on the quality of the measurements. In this paper, we assume that the scans will be registered using the adjustment of surface correspondence, such as the method proposed by Gruen and Akca (2005).

Ideally, the solution of this problem would allow to scan and cover the entire scene from a predefined number of view points. The determination of the positions of the view points to scan all the visible surfaces is a well-studied problem in computer and machine vision, known as the Art Gallery problem (Chvatal, 1975; O'Rourke, 1987) or the Next Best View problem (Reed and Allen, 1999; de Berg et al., 2008). Generally, these algorithms determine the next location to place the scanner to maximize the amount of information to be captured that will be added to the already present one. These algorithms generally assume the sensing instrument to be perfect, with an infinite field of view, with precise point measurements under any incidence angle or range constraints and with almost instant acquisition times. These solutions are very general and are often unsuitable for the actual scanner capabilities in a real world situation. In practice, the scanner has range limitations set by the manufacturer, both with a minimum distance and a maximum distance of measurements of a surface. Furthermore, it has been shown in Soudarissanane et al. (2011) that high incidence angles deteriorate the quality of measurements, therefore a constraint on incidence angles is added to ensure a good quality of acquisition.

To significantly reduce the number of scans to cover an entire scene and ensuring good quality scans, we propose an optimal view point localization method based on a map of the area and scanner constraints. This method comprises the following constraints:

- The map is a horizontal cross-section obtained at the height of the scanner. This 2D map may include holes,

- The scanner has a range limitations defined by the manufacturer,

- The incidence angles measured on surfaces should not exceed a defined threshold.

To solve this optimization problem under the above mentioned constrains, the following requirements should be fulfilled:

1 - All edges of the 2D map are covered by at least one view point

2- All edges are covered from at least one view point with at least a minimum distance $d_{\min }$ and with a maximum distance $d_{\max }$ to the scanner

3 - All edges are scanned from at least one view point with an incidence angles below a maximum incidence angle $\alpha$.

To define the optimal view points, the interior of the scene needs to be sampled with potential view point locations. The room is discretized and gridded with predefined steps of possible scanner locations, as depicted as an example in Fig. 3(a). This discretization has the advantage of less computations than considering continuous locations. However, the grid step size needs to be well defined to avoid any conflicts with the set constraints, i.e. minimum distance $d_{\min }$ and maximum distance $d_{\max }$. Too large grid will result in very fast computations, but less accurate optimal positions. Too small grid increase the processing time with no guarantee of having a better location.

\subsection{D Visibility Polygon}

The scene is defined as a 2D map consisting of a simple outer bound polygon $\mathbf{P}_{\mathbf{o}}$ and $K$ simple holes polygons $\mathbf{P}_{\mathbf{h} j}, j=1 \ldots K$. As defined by de Berg et al. (2008), a simple polygon is a closed polygonal chain of line segments that does not intersect itself. The interior and the exterior of a simple polygon is well-defined. Holes are interior obstructions caused by e.g. walls, furniture in the room, columns.

To define potential view point locations, the interior of the room is sampled such that a view point $O_{i}$ is defined inside $\mathbf{P}_{\mathbf{o}}$ and outside all $\mathbf{P}_{\mathbf{h} j}$. This room is gridded with $n$ possible view point locations, denoted as $O_{i}, i=1 \ldots n$. The grid step is defined so that each edge part can be visible from at least one view point.

The definition of the 2D visibility polygon $V_{i}$ from the view point $O_{i}$ is a well-studied problem (Erdem and Sclaroff, 2006; Landa et al., 2006; Obermeyer et al., 2010; Tomas et al., 2006). In this paper, the method described by Erdem and Sclaroff (2006) and implemented by Obermeyer and Contributors (2008) is used to determine the $2 \mathrm{D}$ visibility polygon $V_{i}$ from each possible view point $O_{i}$, as depicted in Fig. 1. In this paper, the coverage of the wall surfaces is considered, therefore the polygon edges should all be covered by at least one view point, the complete coverage of the interior of the polygon is not necessary.

\subsection{TLS placement algorithm: Greedy approach}

The definition of the minimum number of view points needed to cover all edges of a room is known as the set-coverage problem and it is a NP-complete problem (Karp, 2010). However, by using a Greedy approach (Chvatal, 1979), the set-coverage algorithm can be implemented to run faster with less computation steps (Slavk, 1996).

Each edge of the room is discretized into smaller intervals to gain in computational performances.For instance, an edge $m$ of the 


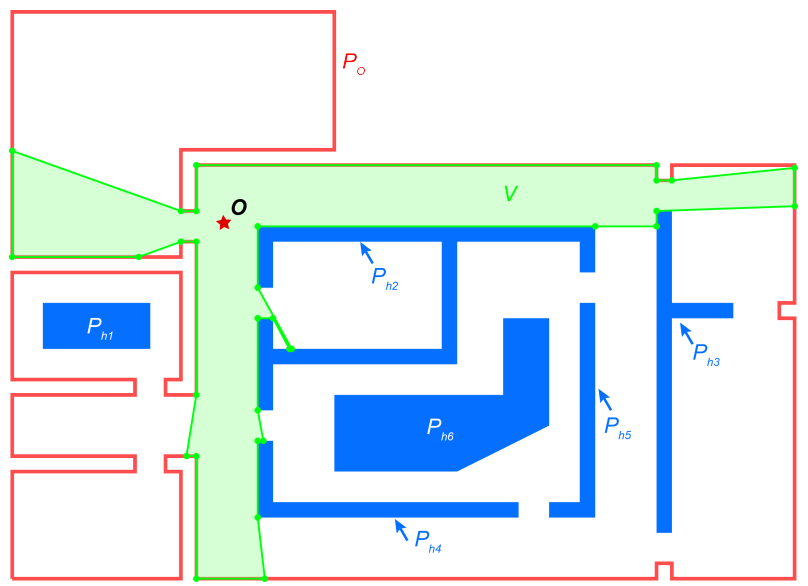

Figure 1: Example of visibility polygon of a complex room. The outer bound polygon $\mathbf{P}_{\mathbf{o}}$ is outlined in red. Interior obstruction polygons $\mathbf{P}_{\mathbf{h} j}, j=1 \ldots 6$ are represented as blue areas. The interior of these polygons is not visible. The view point $O$ is depicted as the red star. The visibility polygon $V$ from this location $O$ is represented as the green area.

outer polygon $P_{o}$ would be discretized into $k$ smaller segments with the interval step $\Delta$ following Eq. 1:

$$
P_{o}\{m\}=\left[\begin{array}{c}
P_{o}\{m\}\{1\} \\
\vdots \\
P_{o}\{m\}\{k\}
\end{array}\right]=\left[\begin{array}{cc}
\{x, y\}_{\text {start }}, & \{x, y\}_{\Delta} \\
\vdots & \vdots \\
\{x, y\}_{(k-1) \Delta}, & \{x, y\}_{\text {end }}
\end{array}\right]
$$

With the edges of the room subdivided into smaller segments, it becomes easy to define the discretized visibility polygon from a view point $O_{i}$ by using boolean notations, as depicted in Fig. 2. In practice the visibility edges do not perfectly coincide with the small segments boundaries and results in partially covered segments. In this paper, these partially covered segments are considered as visible.

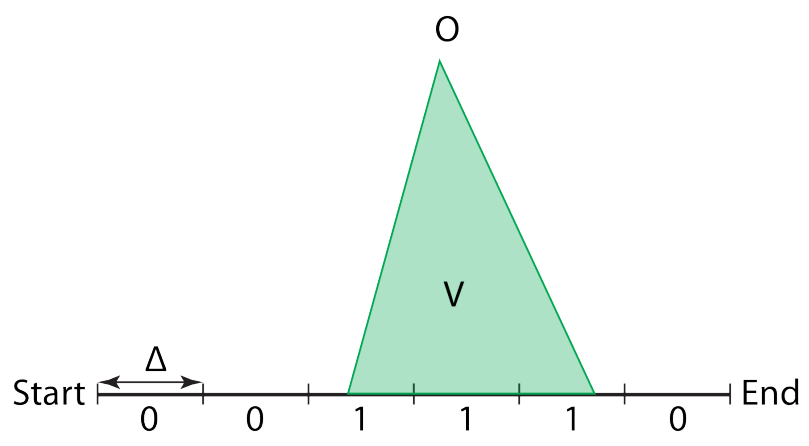

Figure 2: Example of an edge segmentation.

The definition of the interval step $\Delta$ will influence the number of segments $k$ and therefore the precision of the visibility coverage. Too large segments will result in less precise approximations of coverage, but with faster processing due to a low number of segments. On the contrary, a fine segmentation enables to precisely define the boundaries of the visibility coverage at the expenses of longer processing time.

For the $n$ possible view points $O_{i}, i=1 \ldots n$, the $n$ visibility polygons $V_{i}, i=1 \ldots n$ are computed following the implementation of Obermeyer and Contributors (2008). Each visibility polygon is discretized and snapped to the smaller edge segments of the room. The Boolean coverage information per view point is then used in a Greedy algorithm to determine a minimum number of view points necessary to cover all the edges of the room. At each step, this algorithm chooses the view point from which the largest number of segments is covered. The Greedy algorithm produces a good solution, often near the optimal solution, with a faster computation time (Slavk, 1996).

\subsection{Incidence angle and Range constraint}

The Terrestrial Laser Scanner measures distances to surfaces by emitting laser beams, either by continuous modulated signals or single pulses. In both cases, this technology has limitations in the range of measurements, both as a minimum distance $d_{\text {min }}$ and maximum distance $d_{\max }$ that can be measured to an object surface. The manufacturer provides the range limitations specific to each laser scanner type.

Incorporating the range limitations in the determination of the visibility polygon provides a realistic representation of the actual measurements capacity of the scanner (Gonzalez-Banos and Latombe, 2001). Moreover, Soudarissanane et al. (2011) previously showed that the measurement quality deteriorates with bad scanning geometry, i.e. with increasing ranges and increasing incidence angles to the object surface. The incidence angle $\alpha$ is defined as the angle between the laser beam and the normal of the surface. It is shown that increasing incidence angles result in deteriorated signal to noise ratio due to elongated footprints on the surface. Longer ranges result in wider footprints, which are more difficult to detect due to a decrease of signal strength. High incidence angles and longer ranges to the surface therefore result in less precise measurements. Modeling the scanning geometry effects enable to set incidence angles and range constraints to ensure good quality scans.

\section{SIMULATIONS OF MEASUREMENT SET-UP}

To illustrate the method, two simulated case studies are presented. In both cases, a buffer of $d_{\text {min }}=1 \mathrm{~m}$ is applied around all edges of the room to prevent positions too close to the surfaces. The interval step used for the segmentation of the edges is $\Delta=0.05$ $\mathrm{m}$. These test cases are assuming that the scans will be acquired from a mid-range phase based terrestrial scanner, which often has a maximum range limitation of $d_{\max }=80 \mathrm{~m}$. From the study of Soudarissanane et al. (2011), the maximum incidence angle on surfaces is set to $\alpha_{\max }=70^{\circ}$.

The first scene is a simple squared room of $400 \times 400 \mathrm{~m}$, as depicted in Fig. 3. The room is sampled with 361 possible view points spaced every $20 \mathrm{~m}$ on a regular Cartesian grid. This simple test case is chosen to illustrate the impact of the scanning geometry constraints on the definition of the optimal view points locations.

The second scene is a more complex room of $520 \times 380 \mathrm{~m}$, with multiple rooms and occlusions on walls, as depicted in Fig. 4. 356 possible view points are chosen on a regular Cartesian grid. The effects of occlusions is demonstrated in this test case.

\section{RESULTS AND DISCUSSIONS}

By considering only the line of sight of the scanner, the simple room can be completely covered by one view point, depicted in Fig. 3(a). This simple room is a convex polygon, therefore no occlusion are expected from the edges. Any of the possible 361 view points fulfill the complete coverage of the simple room. The complex room is concave. Edges of such a polygon can occlude 
areas in the polygon depending on the view point. As depicted in Fig. 4(a), multiple view points are required to obtain a complete coverage of the scene. In this case, five view points placed in the different opened rooms cover all the edges of the scene.

Applying the incidence angle constraint on the visibility polygons of a view point reduces the field of view of the scanner with respect to the surface orientations. As shown in Fig. 3(b), the simple room can still be fully visible with one view point, under the incidence angle constraint of $\alpha_{\max }=70^{\circ}$. This constraint does not change the number of view point, it however provides a better position of the scanner from which incidence angles on surfaces are tolerable. As the complex room contains more edges with different orientations, the number of view points required to cover all the edges with an incidence angle smaller than $\alpha_{\max }$ doubles to ten view points, as seen in Fig. 4(b).

The range constraints of $d_{\min }=1 \mathrm{~m}$ and $d_{\max }=80 \mathrm{~m}$ enforce a realistic representation of the measurements capabilities of a terrestrial laser scanner. The coverage of all edges of large rooms under range constraints results in the increase of the number of view points necessary to cover the scene. In the simple room case, twelve view points are needed, as shown in Fig. 3(c). As opposed to the visibility coverage without any constraints, many view points are required to cover a complete single edge of a scene. Fig. 4(c) shows that the complex room is covered by seventeen view points, which is a similar number as the ones required under incidence angle constraint. This small difference is explained by the smaller ranges of the small opened rooms, which do not require more view points to fulfill the range constraints.

By incorporating both the maximum incidence angle $\alpha_{\max }$ and the range constraints $d_{\min }$ and $d_{\max }$, the optimal view points necessary to cover a large room can be defined. These optimal view points ensure low incidence angle on surface and measured ranges to the surfaces that are within the specifications of the in strument. The effects of bad scanning geometry are avoided, resulting in more reliable point clouds. As depicted in Fig. 3(d), fifteen view points are necessary to cover the simple squared room. Nineteen view points are required to cover the complex room under the range and incidence angle constraints, as seen in Fig. 4(d).

\section{CONCLUSIONS AND FUTURE WORK}

An original method is presented in this paper to determine optimal view points in a scene based on terrestrial laser scanner capabilities. It is shown that by considering the scanning geometry influences in a survey planning, the positions of the view points can be on forehand localized to capture scans of the surroundings with good quality.

The influence of an incidence angle constraint and of a range limitation is shown on two simulated rooms. The presented work uses a Cartesian griding of the room to determine possible view point location. An adaptive grid to the level of detail required per surface would improve the determination of the best view points. Moreover, a finer grid would avoid view points positions too close to each other, however it will be at the expenses of longer processing times.

The presented method handles the incidence constraint and the range constraints as two uncorrelated factors. However, in practices these constraints are closely related. A combined constraint will provide a visibility from a view point that is more realistic.

Currently, the presented method uses a 2D discretized map of the scene at the height of the laser scanner. A possible extension of this method would be to consider the 3D room and define the $3 \mathrm{D}$ visibility from a view point. The integration of several scanner height would improve the determination of the optimal view points.

Finally, in this paper we used a Greedy approach to select the best view points necessary to cover the scene. A better optimization algorithm would provide more optimal view points and better distributed in the scene.

\section{References}

Besl, P. J. and McKay, N. D., 1992. A method for registration of 3-d shapes. IEEE Trans. Pattern Anal. Mach. Intell. 14(2), pp. 239-256.

Borah, D. K. and Voelz, D. G., 2007. Estimation of Laser beam pointing parameters in the presence of atmospheric turbulence. Applied Optics 46(23), pp. 6010-6018.

Bucksch, A., Lindenbergh, R. and van Ree, J., 2007. Error budget of Terrestrial Laser Scanning: Influence of the intensity remission on the scan quality. 1(Part 2), pp. 113-122.

Chvatal, V., 1975. A combinatorial theorem in plane geometry. Journal of Combinatorial Theory 18(1), pp. 39-41.

Chvatal, V., 1979. A greedy heuristic for the set-covering problem. Mathematics of Operations Research 4(3), pp. 233-235.

de Berg, M., Cheong, O., van Kreveld, M. and Overmars, M., 2008. Computational Geometry: Algorithms and Applications. Vol. 3rd rev, Springer-Verlag.

Dorninger, P., Nothegger, C., Pfeifer, N. and Molnar, G., 2008. On-the-job detection and correction of systematic cyclic distance measurement errors of terrestrial laser scanners. Journal of Applied Geodesy 2(4), pp. 191-204.

Erdem, U. M. and Sclaroff, S., 2006. Automated camera layout to satisfy task-specific and floor plan-specific coverage requirements. Computer Vision and Image Understanding 103(3), pp. 156-169.

Faro, 2010. Laser Scanner Photon 120. http://www.faro.com/ (Accessed 10 January, 2011).

Gonzalez-Banos, H. and Latombe, J.-C., 2001. A randomized art-gallery algorithm for sensor placement. (Part 1), pp. 232240.

Gorte, B., Khoshelham, K. and Verbree, E., n.d. Indoor navigation by using segmentation of range images obtained by Laser Scanners.

Gruen, A. and Akca, D., 2005. Least squares 3D surface and curve matching. ISPRS Journal of Photogrammetry and Remote Sensing 59(3), pp. 151-174.

Hejbudzka, K., Lindenbergh, R., Soudarissanane, S. and Humme, A., 2010. Influence of atmospheric conditions on the range distance and number of returned points in leica scanstation 2 point clouds. XXXVIII(Part 5), pp. 282-287.

Hoefle, B. and Pfeifer, N., 2007. Correction of Laser Scanning intensity data: Data and model-driven approaches. ISPRS Journal of Photogrammetry and Remote Sensing 62(6), pp. 415433. 


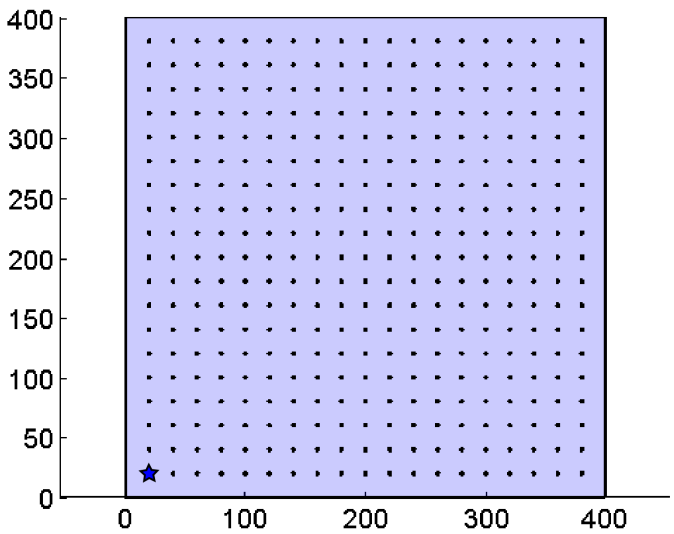

(a) In this case, only one view point is needed. Any of the possible 361 view points fulfill the visibility constraint. The visibility polygon is represented as the blue area.

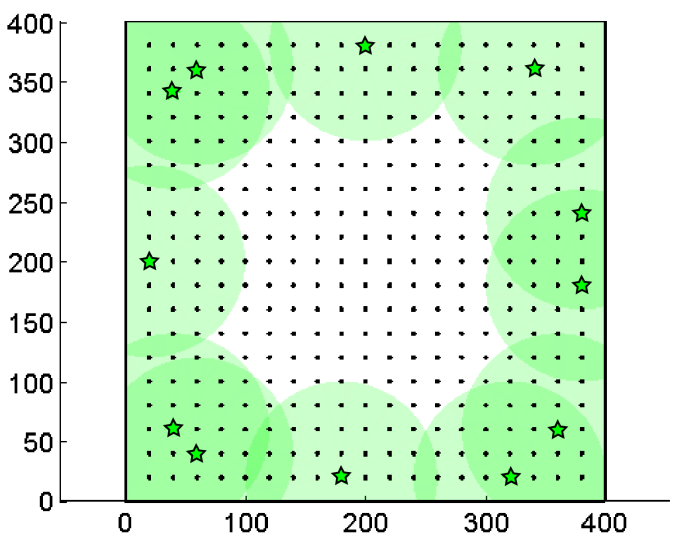

(c) At least twelve view points are required to cover all the edges under range constraints. The visibility polygons are represented as the green areas.

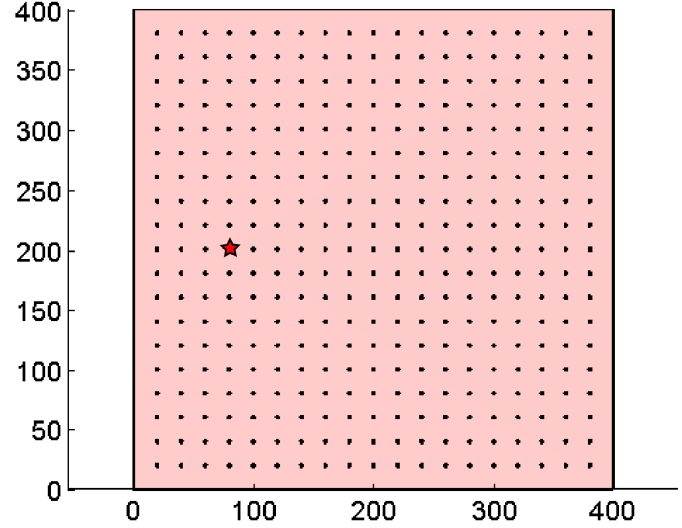

(b) One view point is required to cover all the edges under incidence angles constraints. Multiple view points can fulfill the visibility under incidence angle constraints. The visibility polygon is represented as the red area.

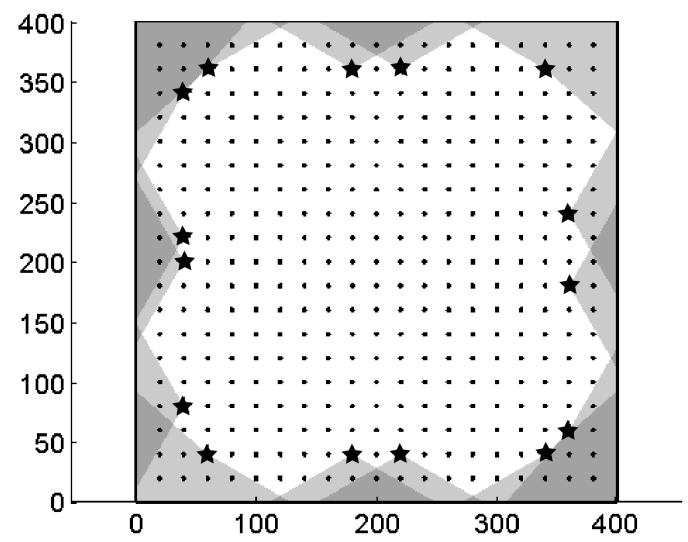

(d) At least fifteen view points are required to cover all the edges under range and incidence angles constraints. The visibility polygons are represented as the gray areas.

Figure 3: Visibility coverage of simple room. 361 view points are used in the scene, depicted as black dots. Possible view points that fulfill the visibility constraints are depicted as stars, with their visibility coverage area. The darker the visibility area, the more view points coverage.

Kaasalainen, S., Krooks, A., Kukko, A. and Kaartinen, H., 2009. Radiometric calibration of terrestrial laser scanners with external reference targets. Remote Sensing 1(3), pp. 144-158.

Karp, R. M., 2010. Reducibility Among Combinatorial Problems. Springer Berlin Heidelberg, pp. 219-241.

Kersten, T. P., Mechelke, K., Sterberg, H. and Acevedo Pardo, C., 2004. Terrestrial Laserscanning System Mensi GS100/GS200 - Accuracy tests, experiences and projects at the Hamburg University of Applied Sciences. XXXIV(Part 5/W16), pp. 8.

Khoshelham, K. and Gorte, B., 2009. Registering point clouds of polyhedral buildings to $2 \mathrm{~d}$ maps.

Landa, Y., Tsai, R. and Cheng, L.-T., 2006. Visibility of point clouds and mapping of unknown environments. 1(Part 1), pp. 1014-1025.

Leica, 2010. Laser Scanner HDS6000. http://www.leicageosystems.com/ (Accessed 10 January, 2010).
Lichti, D. D., 2007. Error modelling, calibration and analysis of an AM CW Terrestrial Laser Scanner system. ISPRS Journal of Photogrammetry and Remote Sensing 61(5), pp. 307-324.

Obermeyer, K. J. and Contributors, 2008. The VisiLibity library. http://www.VisiLibity.org R-1.

Obermeyer, K. J., Ganguli, A. and Bullo, F., 2010. Multi-agent deployment for visibility coverage in polygonal environments with holes. International Journal on Robust and Nonlinear Control 00, pp. 1-31.

O'Rourke, J., 1987. Art Gallery Theorems and Algorithms. Oxford University Press, UK.

Reed, M. K. and Allen, P. K., 1999. 3-d modeling from range imagery: An incremental method with a planning component. Image and Vision Computing 17(2), pp. 99-111.

Schaer, P., Skaloud, J., Landtwing, S. and Legat, K., 2007. Accuracy estimation for Laser point cloud including Scanning geometry. 36(Part 5 - W8), pp. on CD ROM. 


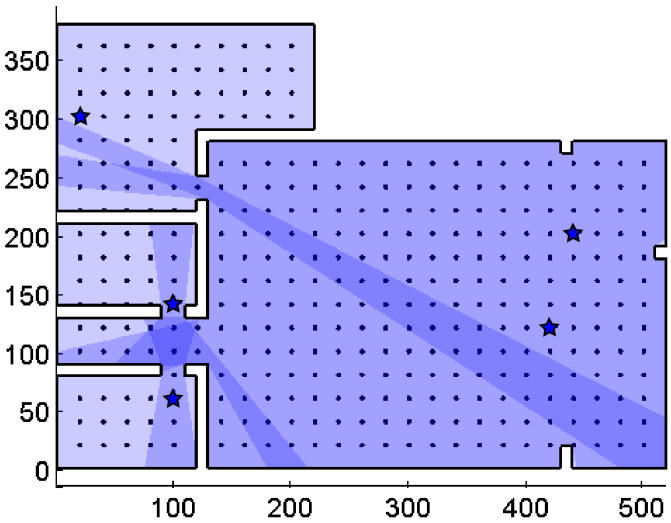

(a) At least five view points are required to cover all the edges. The visibility polygons are represented as the blue areas.

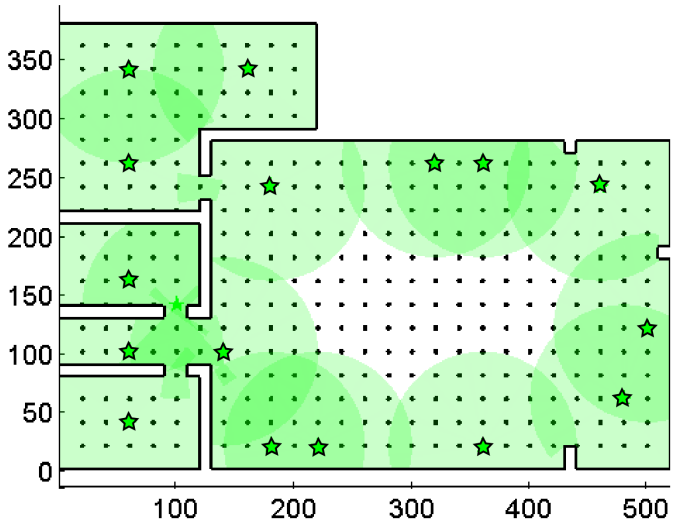

(c) At least seventeen view points are required to cover all the edges under range constraints. The visibility polygons are represented as the green areas.

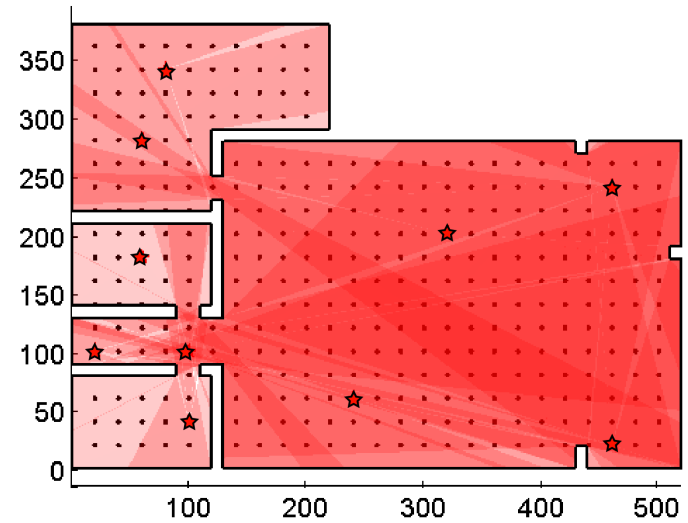

(b) At least ten view points are required to cover all the edges under incidence angles constraints. The visibility polygons are represented as the red areas.

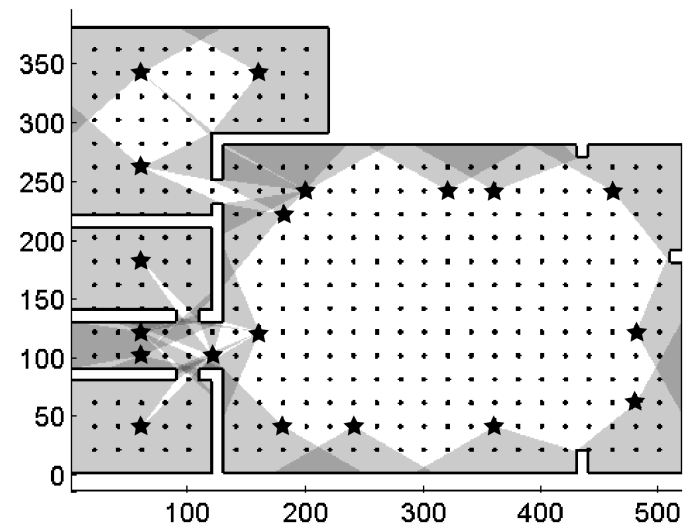

(d) At least nineteen view points are required to cover all the edges under range and incidence angles constraints. The visibility polygons are represented as the gray areas.

Figure 4: Visibility coverage of a complex room. 356 view points are used in the scene, depicted as black dots. Possible locations of the scanner that fulfil the visibility constraints are depicted as stars, with their visibility coverage area. The darker the visibility area, the more view points coverage.

Slavk, P., 1996. A tight analysis of the greedy algorithm for set cover. 1(Part 1), pp. 435-441.

Soudarissanane, S., Lindenbergh, R., Menenti, M. and Teunissen, P., 2011. Scanning geometry: Influencing factor on the quality of terrestrial laser scanning points. ISPRS Journal of Photogrammetry and Remote Sensing.

Tomas, A. P., Bajuelos, A. L. and Marques, F., 2006. On visibility problems in the plane - solving minimum vertex guard problems by successive approximations. 1(Part 1), pp. 1-12.

Voisin, S., Foufou, S., Truchetet, F., Page, D. and Abidi, M., 2007. Study of ambient light influence for three-dimensional scanners based on structured light. Optical Engineering 46(3), pp. 030502-1 030502-3.

Vosselman, G. and Maas, H. G., 2010. Airborne and Terrestrial Laser Scanning. Whittles Publishing, Dunbeath, Scotland, UK.

Vosselman, G., Gorte, B. G. H., Sithole, G. and Rabbani, T., 2004. Recognising structure in Laser scanner point clouds. International Journal of Photogrammetry, Remote Sensing and
Spatial Information Sciences 46(part 8/W2), pp. 33-38. article. 